\title{
Sarcoma de Kaposi clásico con compromiso gastrointestinal multifocal. Reporte de un caso
}

\author{
Andrea Carlin Ronquillo, ${ }^{1}$ Víctor Aguilar Sánchez, ${ }^{1}$ Carlos A García Encinas, ${ }^{1}$ Paul Gómez \\ Hinojosa, ${ }^{1}$ José Luis Pinto Valdivia, ${ }^{1,2}$ Wilmer Silva-Caso ${ }^{3}$ \\ ${ }^{1}$ Hospital Nacional Cayetano Heredia. \\ ${ }^{2}$ Universidad Peruana Cayetano Heredia. \\ ${ }^{3}$ Universidad Peruana de Ciencias Aplicadas. \\ Lima, Perú.
}

Acta Gastroenterol Latinoam 2020;50(3):350-354

Recibido: 16/05/2019 / Aceptado: 07/08/2019 / Publicado online: 28/09/2020

\section{Resumen}

A pesar de que el compromiso intestinal ocurre en más de la mitad de los casos con sarcoma de Kaposi que son VIHpositivos, es poco común en la forma clásica, ya que afecta aproximadamente al $10 \%$ de los pacientes. Presentamos el caso de un paciente varón de 60 años que llevaba un año viviendo con la enfermedad, caracterizado por tener lesiones violáceas en los pies y las manos, ligeramente pruriginosas y con dos meses de epigastralgia, constipación y pérdida de peso de aproximadamente el $12 \%$ de su peso corporal total. En el examen físico se observan múltiples lesiones violáceas del tipo pápula en las manos y los pies, algunas coalescentes formando placas. Los exámenes de laboratorio revelaron una anemia normocítica normocrómica, la serología para hepatitis viral B y C fue negativa y el ELISA VIH también fue negativo. Se le realizó una endoscopía alta y se observaron múltiples lesiones máculo-papulares y eritemato-violáceas en el esófago, el estómago y el duodeno. En la colonoscopía se

Correspondencia: Andrea Carlin Ronquillo Tiziano 1070 (CP. 15037), San Borja. Lima, Perú Tel.: 0051988869184

Correo electrónico: andrea.carlinr@gmail.com reconocieron múltiples lesiones de similares características en el ileon, a lo largo de todo el colon y en el recto. El resultado de la biopsia fue compatible con el SK en todas las lesiones y se confirmó con la inmunohistoquímica $H V V-8$ positiva. Este caso muestra la probabilidad de presentar un sarcoma de Kaposi con un compromiso gastrointestinal y cutáneo en pacientes ancianos VIH negativos, asi como la necesidad de realizar un adecuado diagnóstico mediante la realización de los estudios endoscópicos con las biopsias para un correcto tratamiento.

Palabras claves. Sarcoma de Kaposi visceral, herpesvirus bumano 8.

\section{Classic Kaposi's sarcoma with multifocal gastrointestinal involvement. A case report}

\section{Summary}

Although intestinal involvement occurs in more than half of the cases with KS that are HIV positive, it is uncommon in the classical form, as it occurs in approximately $10 \%$ of the patients. We present the case of a 60-year-old male patient with a one-year disease time characterized by having violaceous lesions on the feet and the hands, slightly pruritic and 2 months of epigastralgia and constipation with weight loss of approximately 12 percent of his total body weight. In the physical examination multiple violaceous papule-like lesions are shown on the hands and the feet, some coalescing to form plaques. Laboratory tests revealed a mild normocytic 
normocytic anemia, the serology for viral hepatitis $B$ and $C$ was negative, HIV negative and ELISA test too. An upper endoscopy was performed and multiple maculopapular and erythematous-violaceous lesions were observed in the esophagus, the stomach and the duodenum. In the colonoscopy, multiple lesions with similar characteristics in the ileum, throughout the colon and in the rectum were recognized. The biopsy result was compatible with the KS in all lesions and it was confirmed with the positive HVV-8 immunohistochemistry. This case highlights the likelihood of presenting GI SK in elderly patients with gastrointestinal compromise and cutaneous findings, HIV negatives as well as the need to realize an adequate discarding by performing endoscopic studies with the biopsies to optimize treatment.

Key words. Visceral Kaposi’' Sarcoma, human herpesvirus 8.

\section{Introducción}

El sarcoma de Kaposi (SK) es un tumor angioproliferativo de bajo grado explicado por el dermatólogo húngaro Moritz Kohn Kaposi en 1872. ${ }^{1,2}$ Más de cien años después, se describe al herpes virus 8 como el posible agente causal. $^{2}$

Existen cuatro variantes: a) SK clásico: suele comprometer a los miembros inferiores. El compromiso visceral es raro, presenta un curso benigno y ocurre sobre todo en los ancianos de ascendencia judía mediterránea, de Europa del Este y askenazí; b) SK africano o endémico: tiene una distribución racial y geográfica específica, así como un curso benigno; c) SK relacionado con la inmunosupresión: ocurre en postrasplantados y en los que reciben la terapia inmunosupresora. Esta forma tiende a ser agresiva y aproximadamente el 50\% tiene afectación visceral; y d) SK asociado al SIDA o epidémico: es la neoplasia maligna más común en estos pacientes. ${ }^{1,2}$

Adicionalmente a la piel, el SK puede presentarse en las mucosas, especialmente en la cavidad oral y en el resto del tracto gastrointestinal (TGI), reportado en el $10 \%$ de pacientes con el SK clásico. ${ }^{2-4}$ La finalidad del presente trabajo es comentar un caso del SK clásico con un compromiso dérmico y la afectación multifocal del TGI.

\section{Caso clínico}

El paciente es un varón de 60 años, procedente de Lima, que tiene lesiones violáceas en los pies y las manos. En un año estas lesiones fueron incrementándose de tamaño, ligeramente pruriginosas. Luego, se asocia a un dolor urente en las plantas de los pies que le dificulta la deambulación. Además, se relaciona a la epigastralgia, la constipación y la pérdida de peso (aproximadamente el $12 \%$ del peso corporal total) que sufre hace dos meses.

En el examen físico se percibe un regular estado general, con funciones vitales estables, sin adenopatías palpables. Se le observan múltiples lesiones violáceas en las manos y los pies, algunas coalescentes, formando placas en las plantas de los pies (Figura 1) y escasas lesiones violáceas en el abdomen.

Figura 1. A) Lesiones violáceas tipo pápula en los pies. B) Algunas coalescentes formando placas en las plantas

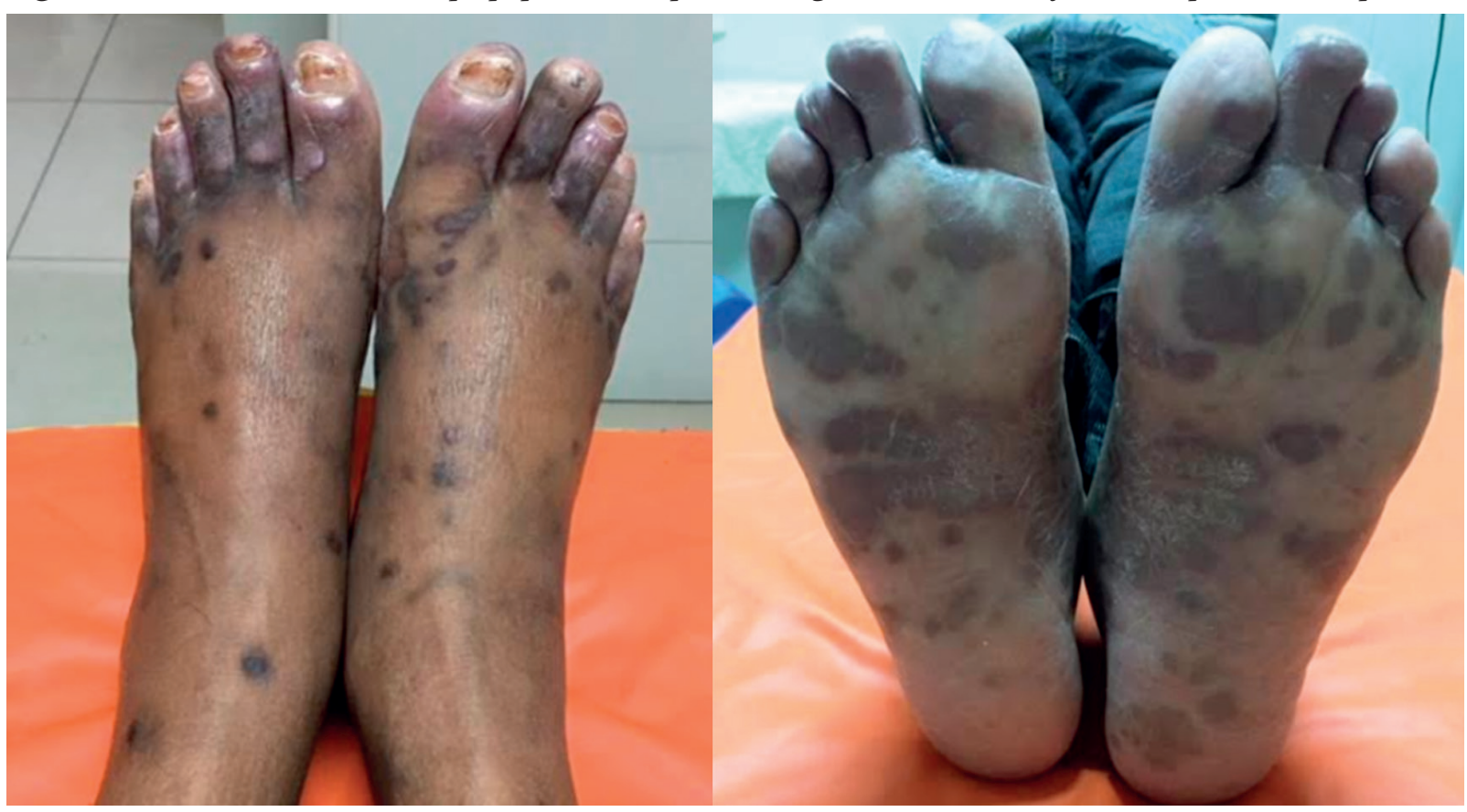


Los exámenes de laboratorio revelan una anemia normocítica normocrómica, la función renal y el perfil hepático dentro de los límites normales, la serología para hepatitis viral B, C y ELISA VIH negativa. Se le realizó una biopsia de las lesiones dérmicas y se le diagnosticó un sarcoma de Kaposi.
Al paciente se le efectuaron estudios endoscópicos: a) videoendoscopía digestiva alta con múltiples lesiones máculo-papulares, eritemato-violáceas en el esófago, el estómago y el duodeno (Figura 2); b) videocolonoscopía con múltiples lesiones de similares características en el íleon, en el colon y el recto (Figura 3).

Figura 2. Videoendoscopía digestiva alta: compromiso gástrico y duodenal. A) Lesiones elevadas rojizas en el cuerpo gástrico con una ulceración central y una tendencia a confluir. B) Lesiones rojizas de aproximadamente 15 mm en el fondo gástrico. $C \boldsymbol{y}$ D) Lesiones rojizas elevadas múltiples que tienden a confluir en la primera $y$ en la segunda porción duodenal

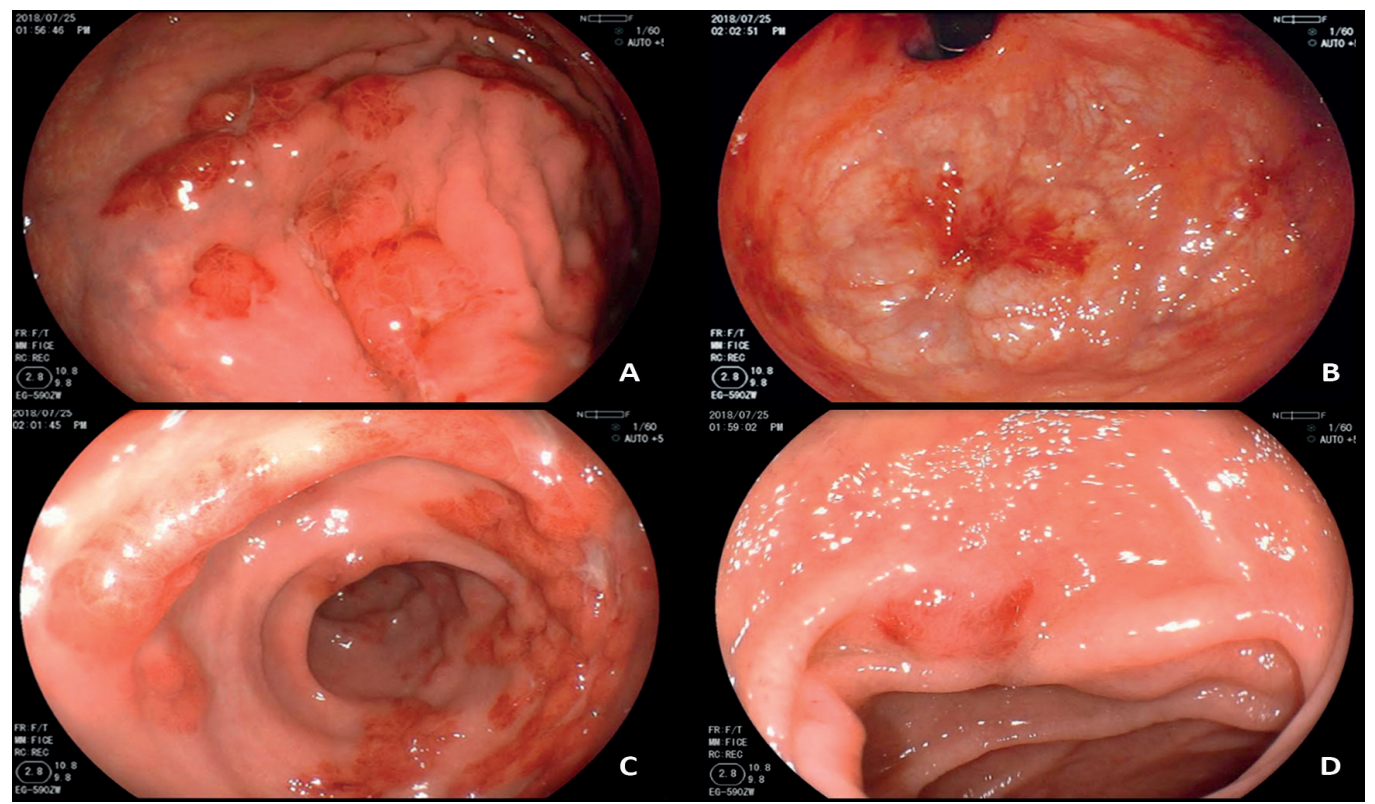

Figura 3. Videocolonoscopía. A, B y C) Compromiso del colon con las lesiones rojizas nodulares umbilicadas. Algunas lesiones rojizas planas en el colon transverso. D) Lesión ileal rojiza de aproximadamente $12 \mathrm{~mm}$ con el centro ulcerado

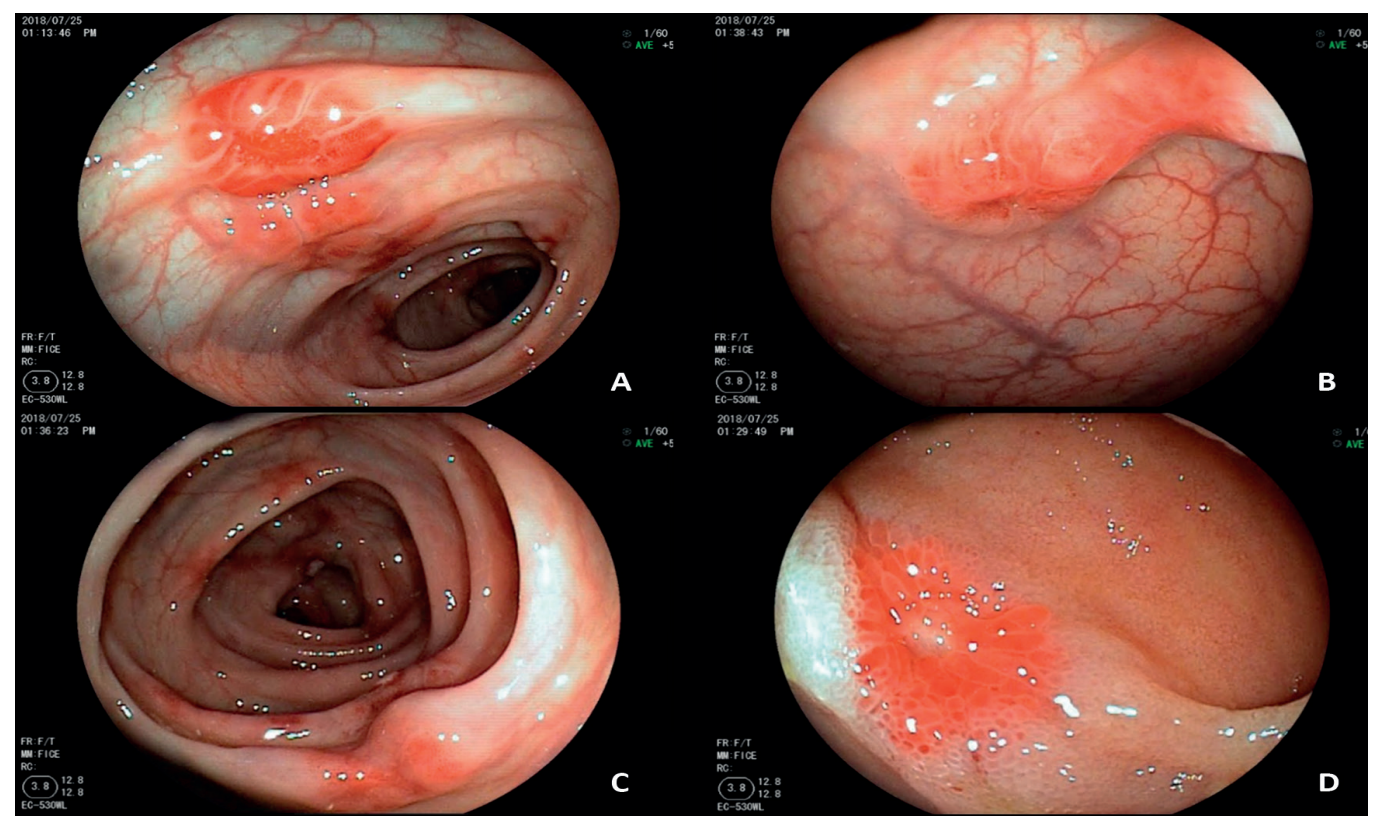


Se tomaron las biopsias de todas las lesiones de las regiones mencionadas cuyo resultado fue compatible con el SK, que se confirmó con la inmunohistoquímica positiva a HVV-8.

\section{Discusión}

Nuestro caso describe a un paciente varón de 60 años de edad con la serología negativa para VIH y para el virus de la hepatitis B y C. Las biopsias de las lesiones dérmicas en las manos y los pies confirmaron el diagnóstico de sarcoma de Kaposi. Además, la inmunohistoquímica de las lesiones gastrointestinales fue positiva a HVV-8. La mayoría de los casos reportados presentan un compromiso cutáneo, con las lesiones predominantemente en los miembros inferiores, como ocurre en el caso descrito. ${ }^{1-3,5}$ Sin embargo, además de las lesiones dérmicas, el paciente manifestó epigastralgia, constipación y pérdida de peso, por lo cual fue sometido a la evaluación por el sector de gastroenterología, donde se determinó la presencia de lesiones a lo largo de todo el tracto gastrointestinal (desde el esófago hasta el recto) con las biopsias reactivas al marcador de herpesvirus- 8 humano. Se sabe que entre el cuarenta al cincuenta por ciento de los pacientes con lesiones cutáneas e infección por VHI tiene una afectación visceral, especialmente en el tracto gastrointestinal (GI-KS), aunque es escasa la información en los pacientes que no presentan infección por VIH., 6

En pacientes con el SK gastrointestinal asociado al SIDA, se describe que la mayoría cursan sin sintomatología digestiva. Algunos presentan síntomas inespecíficos con la progresión de la enfermedad. Entre estos síntomas se pueden mencionar: el dolor abdominal, las náuseas, los vómitos, el sangrado digestivo o relacionado a los cuadros de anemia ferropénica por lo que se requieren exámenes exploratorios. ${ }^{6}$

La presencia del SK, tanto en los pacientes VIH positivos como negativos, puede explicarse desde su fisiopatología relacionada a la expresión del gen tat del VIH-1 que induce el crecimiento de los nódulos. La identificación de algunas citoquinas (la más importante es la oncostatina $\mathrm{M})$ podría indicar que el SK es una neoplasia citoquina inducida. Por otro lado, se describe la teoría sobre la naturaleza infecciosa del SK, debido a la presencia del ADN del virus herpes humano tipo 8 (VHH-8) en las células neoplásicas. ${ }^{2}$ Nuestro caso está relacionado a esta última condición.

El compromiso intestinal es poco común en la forma clásica, ya que ocurre en aproximadamente el 10\% de los pacientes y en la etapa final de la enfermedad. ${ }^{1-3}$ En un estudio en Grecia sobre el SK clásico refieren que el compromiso gastrointestinal involucra principalmente al estómago, al esófago y al duodeno, siendo la mayoría de estas lesiones asintomáticas. ${ }^{2} \mathrm{La}$ afectación colónica es menos común que la del estómago y del intestino delgado. ${ }^{7,9}$ No hay casos reportados aún de un compromiso tan extenso a nivel del TGI como el caso que presentamos.

Las características clínicas que se presentan incluyen la anemia, la hemorragia, la diarrea, la enteropatía perdedora de las proteínas, la perforación y la obstrucción. ${ }^{7-11}$ Los hallazgos endoscópicos son variados y van desde las pápulas discretas, las lesiones volcánicas con umbilicación central a grandes lesiones exofíticas. ${ }^{9}{ }^{12}$

Este caso, así como en la revisión realizada, se muestra la probabilidad de presentar un sarcoma de Kaposi con un compromiso gastrointestinal y cutáneo en pacientes ancianos con VIH negativos. Al mismo tiempo, se señala la necesidad de efectuar un adecuado diagnóstico mediante la realización de los estudios endoscópicos con las biopsias para un correcto tratamiento. ${ }^{12}$

\section{Conflictos de intereses. Los autores declaran no tener nin- gún conflicto de interés.}

\section{Sostén financiero. Los autores declaran no haber recibido apoyo financiero externo.}

\section{Referencias}

1. Carmo J, Chaves Marques S, Bispo M, Pinto D, Chagas C. Clinical and Endoscopic Features of Gastrointestinal Kaposi Sarcoma: A Single-Center Portuguese Experience over the Last Decade. GE Port J Gastroenterol 2017; 24 (5): 219-226.

2. Béjar C, Ayaipoma A, Chián C. Sarcoma de Kaposi clásico. Folia Dermatol Peru 2008; 19 (1): 27-31.

3. Mohanna S, Maco V, Bravo F, Gotuzzo E. Epidemiology and clinical characteristics of classic Kaposi's sarcoma, seroprevalence, and variants of human herpesvirus 8 in South America: a critical review of an old disease. Int J Infect Dis 2005; 9 (5): 239-250.

4. Paredes-Pacheco E, Albújar P. Sarcoma de Kaposi: Comunicación de un caso. Revista del Cuerpo Médico (Hospital Obrero de Lima) 1968; 7: 451-454.

5. Mohanna S, Maco V, Gown AM, Morales D, Bravo FG, Gotuzzo E. Is classic Kaposi's sarcoma endemic in Peru? Report of a case in an indigenous patient. Am J Trop Med Hyg 2006; 75 (2): 324-326.

6. Le Mouel JP, Chatelain D, Hautefeuille V. Gastrointestinal: Disseminated Kaposi's sarcoma. J Gastroenterol Hepatol 2018; 33 (7): 1309.

7. Mohanna S, Sánchez J, Ferrufino JC, Bravo F, Gotuzzo E. Sarcoma de Kaposi Clásico en Yeyuno: Reporte de Caso. Rev Gastroenterol Perú 2005; 25 (3): 285-287. 
8. Tas F, Keskin S, Yegen G, Gozubuyukoglu N. Classic Kaposi's sarcoma with colonic involvement: A rare presentation with successful treatment with oral etoposide. J Can Res Ther 2012; 8 (1): 112-113.

9. Balachandra B, Tunitsky E, Dawood S, Hings I, Marcus VA. Classic Kaposi's sarcoma presenting first with gastrointestinal tract involvement in a HIV-negative Inuit male -- a case report and review of the literature. Pathol Res Pract 2006; 202 (8): 623-626.
10. Novis BH, King H, Bank S. Kaposi's Sarcoma Presenting with Diarrhea and Protein-Losing Enteropathy. Gastroenterology 1974; 67 (5): 996-1000.

11. 'Aprile M, Di Blasi A. Protein-losing enteropathy in a patient with generalized Kaposi's sarcoma. Cancer 1981; 47 (3): 588-591.

12. Jiménez K, Martínez J, Hernández G, Garzón M, Hormaza N, Lizarazo JI, Marulanda, JC, Molano JC, Rey M, Gómez Zuleta M. Tres variedades de sarcoma de Kaposi con compromiso gastrointestinal. Rev Col Gastroenterol 2015; 30 (3): 342-346. 